\title{
Use of dermal matrices to change gingival phenotypes.
}

\author{
Javier Rojas ${ }^{1 *}$, Leonardo Righesso², Leticia Rojas ${ }^{3,4,5}$
}

\author{
1. Private practice. Clínica Dr. Nicolas Troncoso. La \\ Dehesa, Santiago, Chile. \\ 2. PhD Candidate. Clinic for Oral and Maxillofacial \\ Surgery and Plastic Surgery, University Medical \\ Center of the Johannes Gutenberg University. \\ Mainz, Germany. \\ 3. PhD Candidate. Translational Allergy and \\ Immunology Laboratory, Faculty of Medicine, \\ Pontificia Universidad Católica de Chile, Santiago, \\ Chile. \\ 4. Periodontal Biology Laboratory, Faculty of \\ Dentistry, Universidad de Chile, Santiago, Chile. \\ 5. Oral Pathology Laboratory, Faculty of Dentistry, \\ Universidad Andrés Bello, Santiago, Chile. \\ * Correspondence Author: Javier Patricio Rojas \\ Cortez | Address: Marchant Pereira 2950, \\ Depto.306. Ñuñoa. Santiago. Chile | Phone: +56 9 \\ 33406705 | E-mail: dr.javierrojasc@gmail.com \\ Work received on 17/03/2020. \\ Approved for publication on 25/05/2020
}

\begin{abstract}
A variety of periodontal plastic surgery techniques have been proposed to correct aesthetic and functional problems of periodontal tissues. The most common procedure uses the connective tissue graft from the palate. However, patient discomfort and the limited quantity of palatal tissue results in many cases where the surgeon uses connective tissue substitutes. In this case report, we describe the use of a human dermal matrix for gingival volume augmentation in the lower incisors with a novel modified VISTA technique stabilizing the flap with sutures and cyanoacrylates, avoiding comorbidity of a second surgical site. The follow_up at 6 months showed an increase in gingival thickness and a reduction in recessions length..
\end{abstract}

KEY WORDS:

Gingival augmentation; Dermal matrix; Connective tissue graft.

Int. J. Inter. Dent Vol. 13(2); 99-101, 2020.

\section{INTRODUCTION}

Periodontal plastic surgery is defined as the surgical procedure that aims to prevent, correct and eliminate developmental or traumatic deformities of the gingiva and alveolar mucosa. Among the objectives of the procedure are recovering the morphology, position and dimension of the gum surrounding the tooth or dental implant ${ }^{(1)}$.

One of the most common indications of periodontal plastic surgery is the treatment of gingival recession and the gingival phenotype modification $^{(1,2)}$.

Its etiology is determined by a series of factors such as a lack of attached gingiva, thin gingival phenotype, inadequate dental position, radicular prominence, bone dehiscence, inflammation, traumatic brushing, gingival laceration, inadequate design of removable prosthesis, uncontrolled orthodontic tooth movements and high frenum insertion that facilitate traction towards the apical gingival margin during movements ${ }^{(1)}$.

The indication of root coverage and gingival volume augmentation surgeries contemplate the presence of aesthetic problems, sensitivity or susceptibility to root caries, lack of attached gingiva and cervical abrasion $^{(3)}$.

Multiple surgical modalities have been used to achieve a correct and functional gingiva in terms of volume and position around the teeth. A solid scientific basis supports the use of subepithelial connective tissue grafts because they have been shown to be highly successful and predictable due to their double source of nutrition ${ }^{(2,3)}$. The use of autogenous soft tissue grafts, however, are principally associated with morbidity at donor sites ${ }^{(1,2,3)}$.

Trying to solve the problem of morbidity and availability of connective tissue is that the acellular dermal matrix (ADM) was developed as an alternative donor material presented to reduce related morbidity and provide unlimited tissue for periodontal surgery ${ }^{(4)}$.

The use of this biomaterial does not require surgery in the donor area and offers advantages over mucosa grafts such as decreased bleeding and postoperative complications ${ }^{(5)}$.

\section{Acellular dermal matrices}

Past surgical techniques for periodontal plastic surgery were developed primarily for subepithelial connective-tissue grafts. While reasonable results can be achieved with those methods, they are not as appropriate for acellular dermal matrix grafts, which involve a different healing process than connective tissue grafting. Although connective tissue and acellular dermal matrix have a slightly different histological appearance, both can successfully be used to increase volume and to cover denuded roots with similar attachments and no adverse healing ${ }^{(6)}$.

The use of such grafts for the oral rehabilitation of patients has greatly broadened the scope of clinical dentistry, with a major benefit to patients being the avoidance of palatal tissue harvesting $(1,2,6)$. Acellular dermal matrix grafts (like Alloderm, OraCell, DermACELL and others) have an advantage over autogenous subepithelial connective tissue grafts in that there is unlimited availability. Using an allograft allows for inclusion of as many sites as necessary in just one surgical procedure, which not only improves patient case acceptance but allows for the treatment of largescale cases not previously possible ${ }^{(2,6)}$.

The surgical procedure is more efficient for the surgeon and less traumatic for the patient and the predictability and long-term success of dermal matrix grafts have been well documented in soft-tissue augmentation around teeth as well as dental implants. The high-quality biomaterial, its natural esthetic appearance, and patients' increased acceptance of therapy make this a desirable replacement procedure for palatal soft-tissue grafting ${ }^{(6)}$.

The manufacturer of a human decellularized dermis material, LifeNet Health, promotes that OrACELL ${ }^{\mathrm{TM}}$ retains native growth factors along with collagen and elastin. Therefore, OrACELL ${ }^{\mathrm{TM}}$ is potentially an excellent candidate for use in the treatment periodontal defects like lack of gingival volume $^{(7)}$. OrACELL undergoes the patented MATRACELL process which includes the following 4 steps: 1) Decellularization with an anionic, nondenaturing detergent, $\mathrm{N}$-Lauroyl sarcosinate to remove donor cells and Benzonase $^{\circledR}$, a recombinant endonuclease, to degrade DNA. 2) Rinsing with water to remove residual reagent and donor cell remnants. 3) Preservation with a solution that is comprised of USP Glycerol and USP Saline to allow for room temperature storage. 4) Sterilization with lowdose gamma irradiation at low temperatures ${ }^{(7)}$.

Regarding the surgical procedure, the VISTA technique is a minimally invasive approach that use a single incision allowing access to the entire surgical region ${ }^{(8)}$. Additionally, it allows better access to the surgical site since it occupies an extensive vertical incision for the instruments to develop a tunnel, decreasing risk of flap perforation, facilitating the positioning and adaptation of the graft. We used a modified VISTA technique that includes smaller multiple remote access points reducing the trauma to the tissues but maintaining the good access to instruments. To stabilize the flap, we combined the use of sutures with tissue adhesives like cyanoacrylates; this combination gives to the flap a double stabilization capacity that is important for the vascularization during wound healing ${ }^{(9)}$.

In the present case report we use ADM as an alternative to connective tissue grafts to augment gingival volume in the lower incisors with a novel modified VISTA technique stabilizing the flap with sutures and cyanoacrylates.

\section{CASE REPORT}

A 43-year-old female patient, ASA I, consulted for discomfort on the gum during toothbrushing. At the clinical examination, a recession was 
diagnosed as $R T 2^{(10)}$ with a gingival extension of $2 \mathrm{~mm}$ (fig. 1) and $1 \mathrm{~mm}$ of gingival thickness, measured with periodontal probe (North Carolina, American Eagle, USA). The characteristics of the gingival margin, gingival thickness and dental morphology are compatible with thin phenotype. Scaling and hygiene instruction were performed one week before surgery. The objective of the surgery was to increase the gingival thickness to develop a thick phenotype. The patient was informed about the benefits and risks of the procedure and signed an informed consent.

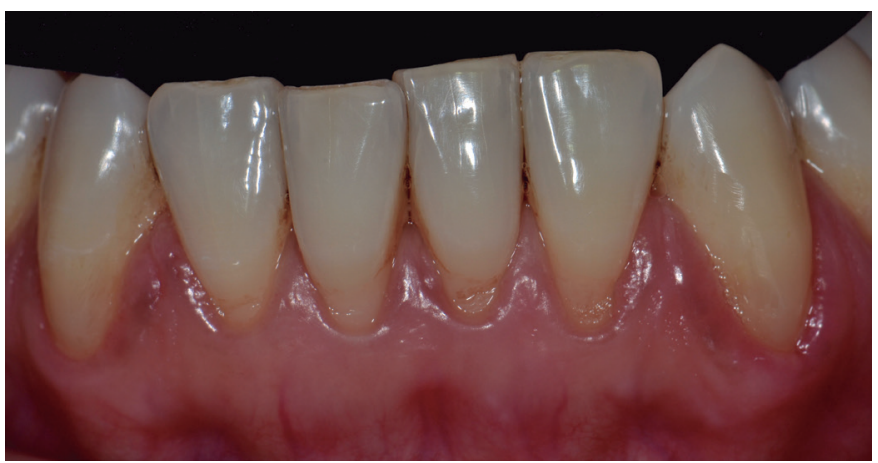

Figure 1. Baseline recessions: The recessions class RT2 are from 2 to $3 \mathrm{~mm}$ length. The phenotype was classified as thin. In 3.2 it can be seen the translucency of the root.

The VISTA technique is used as the basis for this procedure. Different modifications were incorporated such as the absence of a large vestibular access incision and sutures bonded with composite to the teeth. Instead of that, sling suture technique and small holes were made.

During the surgical procedure, $2 \%$ lidocaine was administered with epinephrine 1: 100,000 (Lignospan, Septodont. France). The root scaling of the exposed surface was done with a Gracey curette Sub 0 (Medesy, Italy). A full thickness incision was done in relation to the sulcus of the incisors with scalpel No. 15c (Swann - Morton Ltd. Sheffield. England).

Then envelope preparation (receiving site) was carried out by specific instruments for that purpose like the papilla and facial tunneling instrument design by Dr. Sonia Leziy (Dental USA. USA). Subsequently, the flap was moistened using a gauze with physiological saline solution. We made small holes with a needle near the canines and then we introduced via those holes the tunneling instruments to separate the tissue and to develop a full thickness flap (Fig. 2).

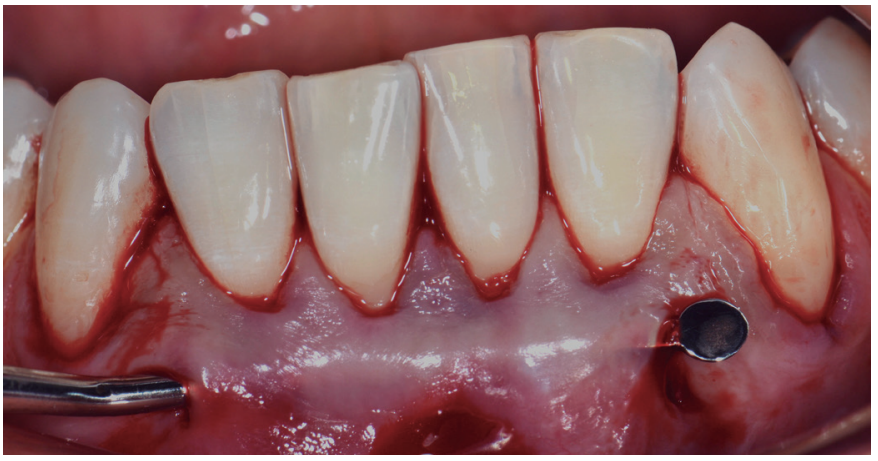

Figure 2. Apical coronal tunnel technique: Via small holes the tunneling instruments were use to separate the tissue and to develop a full thickness flap.

Next, a dermal matrix (OrACELL ${ }^{\mathrm{TM}}$, LifeNet Health, USA) of human origin measuring $1.5 \mathrm{~mm}$ thick and $4 \mathrm{~cm}$ long was used. According to the manufacturer, once the package is opened, this dermal matrix is ready to use and does not require prior hydration, since it comes embedded in a saline solution and glycerol. It was cut in half to fit the length of the area where the increase in gingival volume was required. The dermal matrix was put in place via a hole in the gum (Fig.3). The flap and matrix were stabilized using sling suture technique with 6-0 braided synthetic absorbable suture (Polysorb ${ }^{\mathrm{TM}}$, Covidien, USA).

Finally, 2 layers of tissue adhesive were applied (PeriAcryl $90 \mathrm{HV}$, GluStitch Inc., Canada) until a compact and stable surgical dressing was achieved that protects and stabilized the surgical area for 10 days (Fig. 4). Full surgical time of the procedure was 41 minutes.

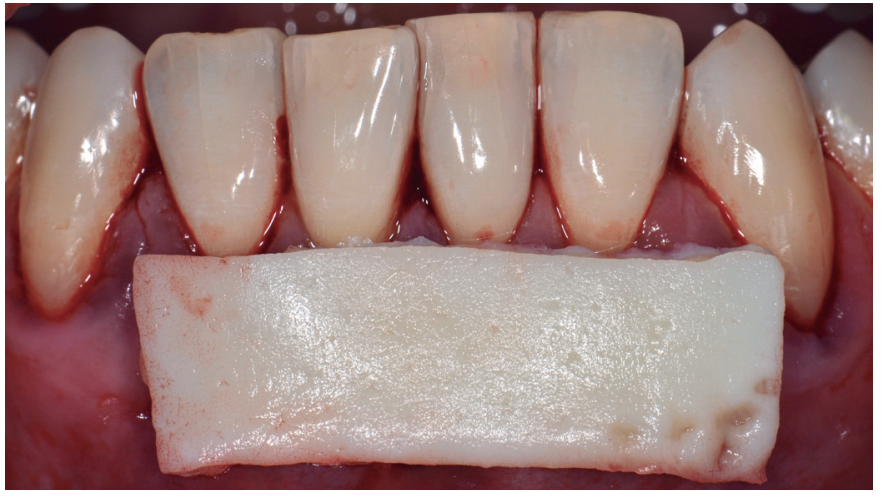

Figure 3. Dermal matrix placement: We used an acellular dermal matrix (OrACell, LifeNet. USA) of $1.5 \mathrm{~mm}$ thick and $18 \mathrm{~mm}$ long to cover the incisors region.

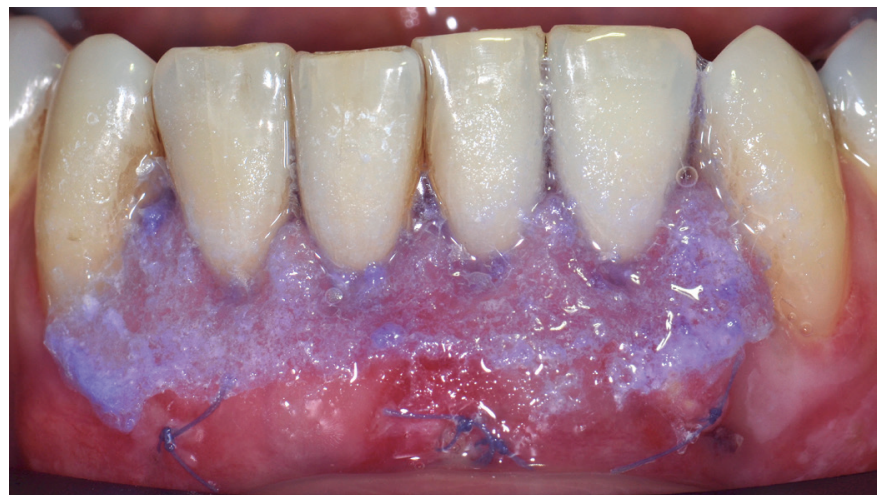

Figure 4. Flap stabilization: Layers of a tissue adhesive (PeriAcryl $90 \mathrm{HV}$ GluStitch. Canada) were used to protect and immobilize the surgical area.

Within the post operative care, the patient was given a "soft" diet for 7 days, plenty of liquid, told not to brush the area for 4 weeks, use clorhexidine for 14 days and lbuprofen $600 \mathrm{mg}$ every 8 hours for 3 days (Actron, Bayer, Germany). The follow up was performed at 7, 14, 90 and 180 days post surgery (Fig. 5a, 5b). At 6 months it was observed a reduction in gingival recession of $1 \mathrm{~mm}$ and an increased gingival thickness of $2 \mathrm{~mm}$ approximately, measured with periodontal probe (North Carolina, American Eagle, USA). Patient reported a complete resolution of the discomfort during toothbrushing.

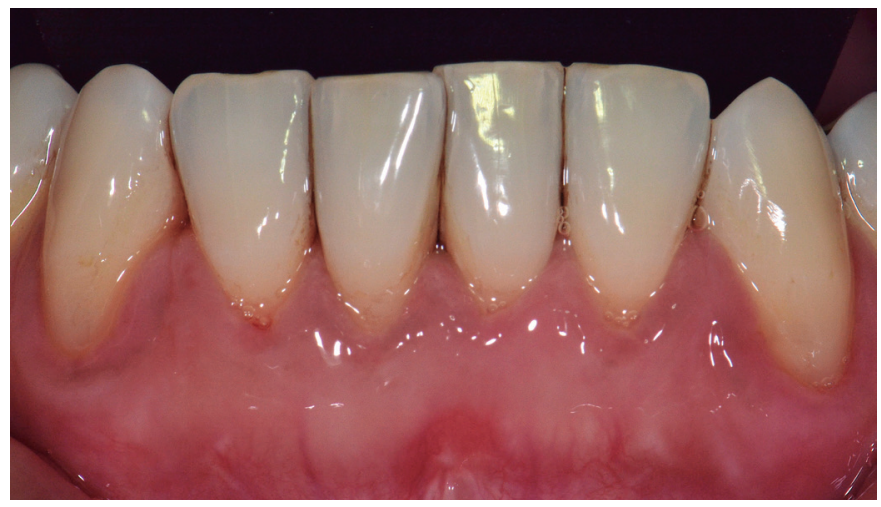

Figure 5. a) Buccal view after 6 months: Follow up 6 months after the surgery a partial coverage of the recessions was achived.

\section{DISCUSSION}

Gingival volume augmentation has become an integral part of periodontal and implant surgery due to the increase in demand for these procedures ${ }^{(10)}$. Periodontal phenotype modification can be performed with different surgical procedures and the available scientific evidence shows that connective tissue graft techniques have the best results in terms of the amount of keratinized gum formed ${ }^{(11)}$. 


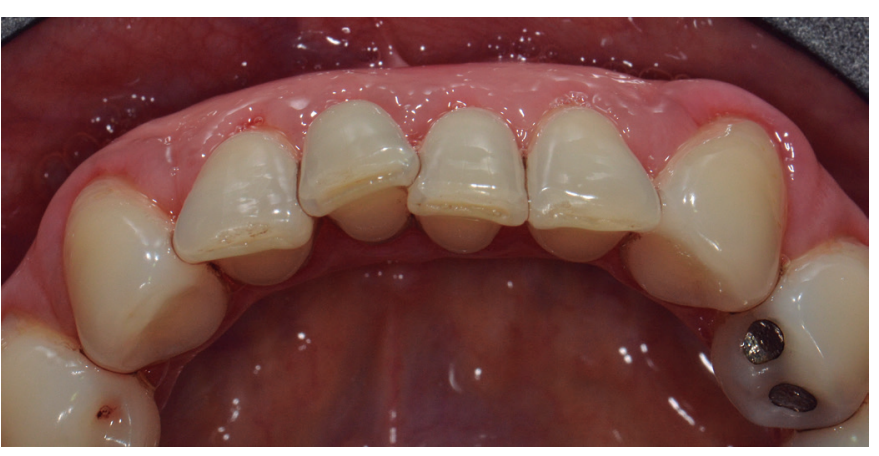

Figure 5. b) Oclussal view after 6 month after surgery:There is a thick gingival phenotype in the incisors region that follow the gingival architecture of the canines.

Connective tissue grafts are considered the standard for gingival volume augmentations to change periodontal phenotypes, as they provide good aesthetic and stable volumetric results. However, the palatal surgical wound produces complications to the patients such as pain, discomfort during feeding, and bleeding ${ }^{(1,2,3)}$.

The use of acellular dermal matrix of human origin arises as an alternative to the palatal connective tissue graft giving a homogeneous thickness, unlimited quantity, avoiding this second surgical intervention, eliminating possible palate complications, reducing patient discomfort and surgical time ${ }^{(7)}$. However, the use of ADM compared to connective tissue has limitations such as decreased creeping attachment, inability to expose, a longer integration period of the matrix in to the tissue, a greater immune reaction associated with more edema and limited formation of attached gingiva $^{(12,13,14,15)}$.

This case report shows the use of human dermal matrix (OrACELL TM, LifeNet, USA) for the modification of periodontal phenotype and reduction of gingival recessions in group $\mathrm{V}$ using a modified VISTA technique, minimizing the access incisions for the instruments that offers less bleeding, trauma, maintaining the direct access to liberate the papillae, allowing less tension in the coronal position flap and reducing perforation risk $^{(8)}$. Additionally, a novel stabilization method of gingival margins using cyanoacrylates was introduced to maintain in combination with sutures the coronal position of the flap during healing. Also, the use of cyanoacrylates works as a surgical dressing, protecting the wound area during the healing period ${ }^{(9)}$.

Reports indicate that in general these procedures have a high learning curve. This can result in a high percentage of graft loss for inexperienced clinicians, high clinical time consumption, complications mainly associated with pain, bleeding, and suture shedding that sometimes leads to failure ${ }^{(10,11)}$. Many of these complications can be avoided by the use of dermal matrices as a substitute for connective tissue ${ }^{(12,13)}$, with a less traumatic surgical technique and a flap stabilizer like PeriAcryl $90 \mathrm{HV}$.

The evidence is inconclusive regarding which graft materials, connective tissue or acellular dermal matrices, develop more keratinized gingiva. Gallagher et al. concludes in a systematic review that it would be the dermal matrix which forms the most keratinized gingiva ${ }^{(15)}$. However, another systematic review from Chambrone et al. would indicate the opposite. Therefore, it is concluded that there would be biases that are not yet elucidated in the literature that can affect the results with one or other material ${ }^{(16)}$.

\section{CONCLUSION}

This paper reports the use of dermal matrices of human origin as an alternative to the use of connective tissue grafts in periodontal phenotype augmentations with 180-day follow-up. We proposed a reliable surgical procedure that includes a modified VISTA technique and a flap stabilization method combining sutures with cyanoacrylates. The evidence of the use of dermal matrices modifying periodontal phenotype is scarce, so it is suggested the development of research on this topic to provide more information on their volumetric stability over time.

\section{CONFLICT OF INTEREST AND FUNDING SOURCE}

Javier Rojas has no conflict of interest and has not received financial support for this article. The project was funded by the author. The dermal matrices used in this study were donated by the company LifeNet, Virginia Beach, Virginia, USA. The other authors have no conflicts of interest.

\section{References}

1. Allen EP. AlloDerm: an effective alternative to palatal donor tissue for treatment of gingival recession. Dent Today. 2006;25(1):48-52.

2. Gapski R, Parks CA, Wang HL. Acellular dermal matrix for mucogingival surgery: a meta analysis. J Periodontol. 2005;76:1814-1822.

3. Mahn DH. Treatment of gingival recession with a modified tunnel technique and an acellular dermal connective tissue allograft. Pract Proced Aesthet Dent 2001;13:69-74

4. Gholami GA, Saberi A, Kadkhodazadeh M, Amid R, Karami D. Comparison of the clinical outcomes of connective tissue and acellular dermal matrix in combination with double papillary flap for root coverage: a 6-month trial. Dent Res J. 2013;10(4):506 513.

5. Hashemi HM, Parhiz A, Ghafari S. Vestibuloplasty: allograft versus mucosal graft. Int J Oral Maxillofac Surg. 2012;41(4):527-530.

6. Gallagher SI, Matthews, DC Acellular dermal matrix and subepithelial connective tissue grafts for root coverage. A systemic review. J Indian Soc Periodontol. 2017;21(6):439-448.

7. Vreeburg SK, Griffiths GR, Rossmann JA. A comparative study of root coverage using Oracle Versus subepithelial connective tissue graft: A randomized controlled trial. Open Dentistry J. 2018;12:977-986.

8. Zadeh $\mathrm{H}$. Minimally invasive treatment of the maxillary anterior gingival recession defects by vestibular incision subperiosteal tunnel access and platelet-derived growth factor BB. Int J Periodontics Restorative Dent. 2011. 31(6):653-660.
9. Tavelli L, Asa'ad F, Raffaele A, Pagni G, Consonni D, Rasperini G. Minimizing patient morbidity following palatal gingival harvesting: a randomized controlled clinical study. International J Periodontics Restorative Dent. 2018. 38:e127-e134.

10. Cairo F, Nieri M, Cincinelli S, Mervelt J, Pagliaro U. The interproximal clinical attachment level to classify gingival recessions and predict root coverage outcomes: an explorative and reliability study. J Clin Periodontol. 2011;38(7):661-666.

11. Vicario - Juan M,Pascual - LaRoccca A, Vives - Bonet MT, Santos - Alemany A. Técnicas de cirugía mucogingival para el recubrimiento radicular. RCOE. 2006;11(1):61-73.

12. Rocuzzo M, Bunino M, Needleman I, Sanz M. Periodontal plastic surgery for treatment of localized gingival recessions: a systematic review. J Clin Periodontol. 2002;29:178-194.

13. Zuchelli G, Mounssif I. Periodontal plastic surgery. Periodontol 2000. 2015;68:333-368.

14. Rocuzzo M, Bunino M, Needleman I, Sanz M. Periodontal plastic surgery for treatment of localized gingival recessions: a systematic review. J Clin Periodontol. 2002;29:178-194.

15. Gallagher SI, Matthews DC. Acellular dermal matrix and subepithelial connective tissue grafts for root coverage: a systematic review. J Indian Soc Periodontol. 2017;21:439-448.

16. Chambrone L, Salinas Ortega MA, Sukekava F, Rotundo R, Kalemaj Z, Buti J et al. Root coverage procedures for treating localized and multiple recession type defects. Cochrane Database Syst Rev. 2018;10(10):CD00761. 